\title{
Protective Effects of Grape Molasses and Resveratrol Against DMBA Induced Oxidative Stress in Rat Ovarian Tissues
}

\author{
Tuğba Raika Kıran ${ }^{1}$ Önder Otlu Ercan Karabulut ${ }^{3}$ Aysun Bay Karabulut ${ }^{4}$ \\ ${ }^{1}$ Iskenderun Technical University Engineering and Nature Science Faculty, Department of Biomedical Engineering, Hatay, Turkey \\ ${ }^{2}$ Turgut Ozal University, Faculty of Agriculture, Department of Soil Science and Plant Nutrition, Malatya, Turkey \\ ${ }^{3}$ Y1ldırım Beyazıt University, Medical Faculty, Department of Medical Pharmacology, Ankara, Turkey \\ ${ }^{4}$ Y1ldırım Beyazıt University, Medical Faculty, Department of Medical Biochemistry., Ankara, Turkey
}

Received: 22 July 2019, Accepted: 20 August 2019, Published online: 28 August 2019

(C) Ordu University Institute of Health Sciences, Turkey, 2019

\begin{abstract}
Objective: The polyphenolic compound, resveratrol (3, 4', 5-trihydroxystilbene), trans-resveratrol is a natural phytoalexin that exists in many different foods such as grape peel and seed, peanut, mulberry and blueberry. Molasses is a natural food obtained by boiling and concentrating fruit juices known to be rich in minerals such as iron, phosphorus, potassium, sodium, calcium and magnesium, and phosphoric acid, formic acid, organic acids and some vitamins. Polyphenolic compounds are natural antioxidants and are known to have protective effects against tissue damage caused by reactive oxygen species (ROS). In this study, we aimed to investigate the protective effects of grape molasses and resveratrol on ovarian damage induced by 7, 12-dimethylbenz [a] anthracene (DMBA).

Methods: A total of 42 old female Wistar Albino rats, aged 18 weeks were divided into six groups. $10 \mathrm{mg} / \mathrm{kg}$ DMBA was injected in the rats in DMBA group subcutaneously on day 0 and day 7 while grape molasses feed with $20 \%$ grape molasses was given along with DMBA application to the rats in DMBA + grape molasses group. Resveratrol was administered by subcutaneous injection at $10 \mathrm{mg} / \mathrm{kg} / \mathrm{day}$ to the DMBA + Resveratrol group, while only feed with $20 \%$ grape molasses was given to the grape molasses group. Resveratrol was administered by subcutaneous injection at $10 \mathrm{mg} / \mathrm{kg} /$ day to the resveratrol group.

Results: GSH activity between the control group and molasses group, DMBA and DMBA + molasses groups and the control group and the DMBA + grape molasses groups was found statistically significant. Regarding the NO activity, the difference between the DMBA and resveratrol groups, DMBA and DMBA + resveratrol groups and the grape molasses and resveratrol groups was found statistically significant. MDA activity between DMBA + resveratrol and DMBA + grape molasses groups, and DMBA + grape molasses and grape molasses groups was found statistically significant.

Conclusion: Molasses as one of the most important nutrient sources of Eastern and Central Anatolia was observed to decrease ovarian tissue oxidative damage induced with DMBA compared to resveratrol.

Key words: DMBA (7, 12-dimethylbenz [a] antrasen), resveratrol, grape molasses, oxidative stress, ovarian

Suggested Citation: Kiran TR, Otlu O, Karabulut E, Bay Karabulut A. Protective Effects of Grape Molasses and Resveratrol Against DMBA Induced Oxidative Stress in Rat Ovarian Tissues. Middle Black Sea Journal of Health Science, 2019; 5(2):151-159
\end{abstract}

Address for correspondence/reprints:

Tuğba Raika Kıran

Telephone number: +90 (326) 6135600
E-mail: traika.kiran@iste.edu.tr

DOI: $10.19127 / \mathrm{mbsjohs} .595016$ 


\section{Introduction}

Rapid developments in technology, changing lifestyles, environmental pollution and chemicals cause negative impacts on human health and natural resources (Im et al., 2019). Therefore, people are exposed to many carcinogens and mutagenic substances of exogenous origin in their daily lives. This exposure may be air, water, food and soil borne and can transform into toxic compounds as a result of biotransformation reactions as well. Increased duration of exposure to such chemicals can cause irreversible and genetic disorders (David, 1999). Recent studies have demonstrated findings that consumption of green fresh vegetables and fruits protects the organism against toxic effects (Zhao, 1999).

Polycyclic aromatic hydrocarbons (PAH) are three or more aromatic ring compounds originating from different environmental and anthropogenic sources as a result of complete or incomplete combustion of carbon and hydrogen containing organic compounds and pyrolysis processes.

PAHs are carcinogens with tumor initiator, enhancer and promoter properties. PAHs inhibit cellular and humoral immunity (Armstrong et al., 2019). PAHs with low toxicity alone show tumor and mutagenic effect after undergoing metabolic activation. DMBA (dimethylbenz [a] anthracene), a PAH-member with environmental toxic effect, is known as procarcinogen and premutagenic. It is also effective as a teratogen, especially in the adrenal gland and fetal brain, which has been reported to exhibit high cytotoxicity and atherogenic properties in vivo - in vitro. DMBA causes genetic mutation as a result of the binding of intermediates from DMBA metabolism to DNA. It also destroys DNA structure and causes lipid peroxidation. It has been reported to exert a carcinogenic effect by increasing the formation of free radicals such as intracellular hydroxyl and superoxide anion radicals (Giovanni et al., 1980; Gao et al., 2007; Zeweil et al., 2019).

Resveratrol (3, 4', 5-trihydroxystilbene), a subpopulation of stilbenes, is a polyphenolic compound found in grapes, wine, peanuts and blueberries. Resveratrol is a non-flavonoid most intensively found in black grape clusters. Resveratrol is a compound of the phytoalexin group that the grape releases to protect against environmental stress and pathogenic attacks such as thirst, cold weather conditions, fungal infections, ultraviolet rays and ozone. There are many studies reporting that resveratrol presents a number of properties with different mechanisms of action. The inhibitory effect of resveratrol has been mainly associated with anti-xenobiotic ability (De-laLastra and Villegas, 2005; De-la-Lastra and Villegas, 2007).

Reactive oxygen species (ROS) are produced continuously in the body as a result of various metabolic and physiological processes. When the balance between ROS production and natural antioxidant activity deteriorates, a table of "oxidative stress" emerges that can lead to serious cellular damage, premature aging, and even cancer development. As a polyphenol, resveratrol is an antioxidant and free radical scavenger that inhibits reactive oxygen species (ROS) by activating AMPactivated protein kinase. It suppresses cyclooxygenase-2 (COX-2) and lipid peroxidation. Thus, resveratrol shows different pharmacological functions such as antiangiogenic, antioxidant, antitumor and cardio-protective and anticancer (Zadi et al., 2018; Al Fatease et al., 2019; Santos et al., 2019).

Molasses, which is a rich source of energy with organic acids, carbohydrates, minerals and various vitamins, is produced mostly from grapes in our country. Grape molasses contains Thiamin (B1) and Riboflavin (B2) and Niacin (B3) vitamins, Phosphorus (P), Iron (Fe), Copper ( $\mathrm{Cu})$, Zinc $(\mathrm{Zn})$, Potassium (K), Sodium (Na), Magnesium Mg), and Calcium (Ca) minerals (Pharm et al.,2014). It is known that resveratrol in the content of black grape molasses inhibits the formation of reactive oxygen species (ROS) triggered by tumor necrosis factor (TNF), and lipid peroxidation in cells. There are also studies showing that resveratrol reduces tumor progression through the inhibition of cyclooxygenase-2 (Cox 2) as well as antimutagenic, cancer-inhibiting effects (Krishna et al., 2002; Yu et al., 2018; Zheng et al., 2018). There are several studies in the literature about protective effects of grape seeds and grape skin on various cancer types. However, we could not found any study about the effect of grape molasses on ovarian cancer. In the light of these information, the present study was designed to investigate the protective and preventive effect of resveratrol and molasses on DMBA toxicity. The aim of this study was to investigate the effect of resveratrol with grape molasses origin as antioxidant and anticancerogenic on the oxidative stress parameters in resveratrol and rat ovary tissue. 


\section{Methods}

\section{Chemicals, animals and diets}

Wistar albino female rats used in this study were obtained from Inonu University Experimental Animal Production and Research Center. Guidelines of Inonu University Experimental Animal Ethics Committee was complied with during the study period. 18-weeks old female rats weighing $205 \pm 13 \mathrm{~g}$ were kept in standard cages until the day of the experiment. Throughout the experiment, the drinking water was changed daily and the standard cage cleaning was done. The rats are housed in rooms with air conditioner in 24-27 ${ }^{\circ} \mathrm{C}$ room temperature with 12 hours of light and 12 hours of dark. A total of 42 rats were divided into 6 groups and fed with standard pellet food during the experiment except molasses groups. In the power analysis, the number of animals in each group considering $\% 90$ power and 0.05 error margin.

\section{Preparation of Resveratrol DMBA}

In our study, a subcutaneously injected resveratrol mixture was prepared by dissolving 110 $\mathrm{mg}$ resveratrol in $110 \mathrm{ml}$ DMSO, which is known as the solvent of many chemicals. $65 \mathrm{mg}$ of DMBA was applied after being dissolved in $65 \mathrm{ml}$ of sesame oil.

\section{Preparation of Grape Molasses}

Grape picked in the harvest period is cleaned out and the acidity is removed by adding grape marl after the crushing and squeezing process. After the resting and filtering process, the sun darkening process is applied and the molasses is packed. The black grape molasses used in the study was obtained from the Arapgir district of Malatya. Feed containing $20 \%$ molasses was prepared.

\section{Experimental Design}

Control Group $(\mathbf{n}=7)$ : The rats in this group were injected subcutaneously $1 \mathrm{ml}$ each day in a mixture of $20 \mathrm{ml}$ of sesame oil and $30 \mathrm{ml}$ of DMSO.

DMBA Group (n=7): $10 \mathrm{mg} / \mathrm{kg}$ DMBA was injected subcutaneously on day 0 and day 7 .

DMBA + Molasses Group (n=7): $10 \mathrm{mg} / \mathrm{kg}$ DMBA was injected subcutaneously on day 0 and day 7 . Molasses feed with $20 \%$ molasses was given every day.

DMBA + Resveratrol Group $(n=7)$ : Resveratrol was administered by subcutaneous injection at $10 \mathrm{mg} / \mathrm{kg} /$ day. $10 \mathrm{mg} / \mathrm{kg}$ DMBA was injected subcutaneously on day 0 and day 7 .
Molasses Group (n=7): Molasses feed with $20 \%$ molasses was given every day.

Resveratrol Group (n=7): Resveratrol was administered by subcutaneous injection at 10 $\mathrm{mg} / \mathrm{kg} / \mathrm{day}$.

\section{Obtaining Ovarian Tissues and Preparing for Analyzes \\ On the $10^{\text {th }}$ day of the study, rats were sacrificed} under general anesthesia. Ovarian tissues from rats were wrapped in aluminum foil and stored at $-70{ }^{\circ} \mathrm{C}$ in deep freezing until the day when biochemical tests were to be carried out.

\section{Tissue Homogenization and Tampons}

Tissues weighed approximately $200 \mathrm{mg}$ were homogenized at a rate of $16000 \mathrm{rpm}$ by adding $2 \mathrm{ml}$ of Tris - $\mathrm{HCl}$ tampon (pH: 7.0). After homogenization, the tubes were centrifuged at 4000 rpm for 10 minutes at $+4{ }^{\circ} \mathrm{C}$. Supernatants formed after centrifugation were taken into eppendorf tubes and kept in the freezer until the day of operation.

\section{Estimation of Oxidative Stress Markers}

\section{Measurement of Reduced Glutathione}

The absorbance of the yellow colored product resulting from the reaction of total sulphydryl content with Ellman's Reagent (DTNB) was determined by spectrophotometrically measuring at $412 \mathrm{~nm}$ (Tietze, 1969).

\section{Measurement of Lipid Peroxidation}

The measurement of MDA, a lipid peroxidation indicator, is based on the spectrophotometric evaluation of the resultant pink-red color absorbance at $532 \mathrm{~nm}$ as a result of the reaction of MDA in the sample with TBA at $95{ }^{\circ} \mathrm{C}$ (Uchiyama and Mihara, 1978).

\section{Measurement of Nitric Oxide}

NO formed by ambient NOS activity is measured at $545 \mathrm{~nm}$ in a color compound spectrophotometer, which is formed after the reaction with Griess reactivity by being reduced from nitrate to nitrite with cadmium garnets (Cortas and Wakid, 1990). For the standard measurement, the standard graphic was drawn with solutions prepared by a serial dilution of the $10 \mathrm{mmol} / \mathrm{L}$ $\mathrm{NaNO}_{3}$ stock solution $(5-200 \mu \mathrm{M})$. 


\section{Statistical Analysis}

Because of the clinical variables are not normally distributed within groups, non-parametric statistics were used. Descriptive statistics were calculated by median, minimum and maximum. Group comparisons according to GSH, NO and MDA were evaluated by using Kruskal-Wallis Variance Analysis. For pairwise comparisons, Bonferroni adjustment was used. Type-I error rate was taken as $\alpha=0.05$ for statistically significance. SPSS 21 software was used for statistical analyses
(IBM Corp. Released 2012. IBM SPSS Statistics for Windows, Version 21.0. Armonk, NY: IBM Corp.)

\section{Results}

Difference between study groups according to GSH, NO and MDA was statistically significant ( $p<0.001$ for overall comparison). Significance values for pairwise comparisons of these groups were given in Table 1.

Table 1. Difference between study groups according to GSH, NO and MDA ( $<<0.001)$. Different letters $(a, b, c)$ on the colons indicate statistical significance $(\mathrm{p}<0.05)$.

\begin{tabular}{|c|c|c|c|c|}
\hline GROUPS & & $\begin{array}{l}\text { GSH } \\
(\mu \mathrm{mol} / \text { wet tissue })\end{array}$ & $\begin{array}{l}\text { NO } \\
(\mu \mathrm{mol} / \text { wet tissue })\end{array}$ & $\begin{array}{l}\text { MDA } \\
(\mu \mathrm{mol} / \text { wet tissue })\end{array}$ \\
\hline CONTROL & Median & $1.89^{\mathrm{a}, \mathrm{b}}$ & 3.07 & 16.83 \\
\hline DMBA & Median & $1.79^{\mathrm{c}}$ & $4.79^{\mathrm{a}}$ & 22.44 \\
\hline DMBA + RESV. & Median & 1.83 & $4.15^{\mathrm{c}}$ & $19.8^{\mathrm{b}}$ \\
\hline $\begin{array}{l}\text { DMBA + } \\
\text { G.MOLASSES }\end{array}$ & Median & $1.84^{b, c}$ & 4.09 & $19.14^{a, b}$ \\
\hline $\begin{array}{l}\text { GRAPE } \\
\text { MOLASSES }\end{array}$ & Median & $1.85^{\mathrm{a}}$ & $3.32^{\mathrm{b}}$ & $18.15^{\mathrm{a}}$ \\
\hline RESVERATROL & Median & 1.85 & $3.51^{\mathrm{a}, \mathrm{b}, \mathrm{c}}$ & 17.66 \\
\hline Overall comparison & & $\mathrm{p}<0.001$ & $\mathrm{p}<0.001$ & $\mathrm{p}<0.001$ \\
\hline \multirow{3}{*}{ Pairwise comparison } & & ${ }^{\mathrm{a}} \mathrm{p}=0.008$ & ${ }^{\mathrm{a}} \mathrm{p}=0.018$ & ${ }^{\mathrm{a}} \mathrm{p}<0.001$ \\
\hline & & ${ }^{\mathrm{b}} \mathrm{p}<0.001$ & ${ }^{\mathrm{b}} \mathrm{p}=0.022$ & ${ }^{\mathrm{b}} \mathrm{p}=0.039$ \\
\hline & & ${ }^{c} p=0.027$ & ${ }^{c} p=0.004$ & \\
\hline
\end{tabular}

GSH activity between the control group and grape molasses group was found statistically significant $(\mathrm{p}=0.008)$. GSH activity between the DMBA and DMBA + grape molasses groups was found statistically significant $(\mathrm{p}=0.027)$. GSH activity between the control group and the DMBA + grape molasses groups was found statistically significant $(\mathrm{p}<0.001)$.

NO activity between the DMBA and resveratrol groups were found statistically significant $(p=0.018)$ and was found statistically significant between DMBA and DMBA + resveratrol groups $(p=0.004)$. NO activity was found to be significant between the grape molasses and resveratrol groups $(\mathrm{p}=0.022)$.

MDA activity between DMBA + resveratrol and DMBA + grape molasses groups was found statistically significant $(\mathrm{p}=0.039)$. MDA activity between DMBA + grape molasses and grape molasses groups was found statistically significant $(\mathrm{p}<0.001)$.

\section{Discussion}

Various animals are used in many in vivo studies in order to produce cancer models. The most commonly used animals for this purpose are rats. In this study we also used Wistar Albino rats in ovarian toxicity model induced with DMBA.

Recently protective effects of polyphenolic compounds obtained from various diet resources, against oxidative stress resulted from cancer and cancer drugs. Polyphenolic compounds are the most important part of natural plant products with known anti-inflammatory, antimicrobial, anti-alergenic and antioxidant effects (Francischi et al., 2017).

Resveratrol, which is one of the phenolic compounds, and richly found in grapes, wine, peanut and soy, has drawn attraction of scientists 
and medical doctors for many years. Resveratrol is known to have anti-oxidative properties as scavenging reactive oxygen species (ROS) such as hydroxyl, superoxide and metal-induced radicals (Leonard et al., 2003; Truong et al., 2018). Grape molasses is a popular and traditional Turkish food produced in East and Middle Anatolia for long time (Ustun and Tosun, 1997). Grape molasse is mainly produced by concentrating the fruit juice with a soluble dry substance up to $70-80 \%$ (Batu et al., 2013). Although effects of various grape products grape seed, grape skin and grape pomac on oxidative stress have been studied in the literature, there is no any study directly investigating effects of grape molasses. In our study we investigated antioxidant effects of grape molasses in comparison of resveratrol, which is among the polyphenolic compounds found in grape molasses on ovarian cancer induced by DMBA in rats.

In the study by Kim et al., it was determined that grape seed extract was chemo-preventive against DMBA-induced breast cancer in adult rats and genistein exhibited similar activity in N-methyl-Nnitrosourea (MNU) breast cancer rat model, thus, it was concluded that both grape seed and genistein effects were based on diet (Kim et al., 2004). In another study conducted on rats, the anti-tumor effect of polyphenolic fractions isolated from grape seeds was investigated by establishing a two-step carcinogenesis protocol with DMBA and TPA (12O-tetradecanoylphorbol). Extracted polyphenolic fractions have been found to have inhibitory effect on epidermal lipid peroxidation. In the present study we also used DMBA to induce ovarian cancer in rats.

The antioxidant activities of grape and grape seed phenolics have been studied in different models in in vitro systems, and low-density lipoprotein (LDL) has been determined to protect $\mathrm{Cu}^{+2}$ against SIN-1-mediated oxidation producing the oxygen-based radical generator 2, 2-azobis (2amidinopropane) dihydrochloride (AAPH) or peroxynitrite. It has been found to protect spleen cells against DNA damage induced by hydrogen peroxide $\left(\mathrm{H}_{2} \mathrm{O}_{2}\right)$ and reduce oxidative stress in PC12 cells that are stimulated by the addition of $\mathrm{Fe}^{2}$ ${ }^{+}$and t-butyl hydroperoxide (Shafiee et al., 2003; Chanvitayapongs et al., 1997). Anticancer effects of grape antioxidants in in vitro and in vivo models have been studied and have been shown to induce cell cycle blockage and apoptosis in cancer cells, and to inhibit carcinogenesis and cancer progression in rodent models (Aggarwal et al., 2004; Garvin et al., 2006; Ebeler et al., 2002). Male Wistar rats were fed with diets containing cellulose (control) and grape fiber for 4 weeks and GSH: GSSG ratio, GSSG / 2GSH pair redox status, superoxide dismutase (SOD), catalase (CAT) and glutathione peroxidase (GPx), lipid peroxidation (LPO) and apoptosis level were evaluated. In the results obtained, the cytosolic GSH: GSSG ratio increased in the grape fiber diet group, and the decline in apoptosis in relation to diet has been attributed to the modulation of the glutathione redox system and endogenous antioxidant enzymes (Lopez et al., 2010). In another study, a reduction was observed in inhibition of oxidative stress, immunosuppression, reduction of tumor growth, tumor diversity and malignant transformation of papillomas to carcinomas in UVB-induced skin cancer in rats administered with grape seed proanthocyanidin diet supplement (Katiyar, 2008). It was found in another study that grape pomac flour alleviated hepatic oxidative stress induced in carps, by improving antioxidant defence. It was reported that this effect occurred by prevention of overproduced ROS and NOx as well as by prevention of lipid damage (Souza et al., 2019).

A study investigating the effect of grape juice consumption on 7, 12-dimethylbenz [a] anthracene (DMBA)-induced female rat breast tumorigenesis has shown that tumor mass and tumor growth was inhibited. It has been concluded that specific components or phytochemicals in purple grape juice may interfere with the initial phase of DMBAinduced rat breast tumorigenesis (Jung et al., 2006).

In MCF-7 rat cells transfected with aromatase, grape seed extract has been found to inhibit enzyme activity and formation responsible for the transformation of androgens into estrogens and suppress estrogen biosynthesis of procyanidin B2 dimer obtained from grape seed and red wine (Eng et al., 2003; Kijima et al., 2006).

The medium-term oral carcinogenesis process initiated by 4-nitroquinoline 1-oxide (4NQO) was initiated to evaluate anti-tumor activity of grape juice concentrate. As a result of the study, there was a decrease in hyperplastic and dysplastic lesions, a decrease in COX-2 and TNF-alpha and eNOS gene expression, and an increase in $\mathrm{SOD} \mathrm{Cu} / \mathrm{Zn}$ and catalase activity in the grape juice supplement group (Pacheco et al., 2014).

In another study, grape seed pro-anthocyanidins (GSP) was reported to have increased pro-apoptotic Bax protein expression, decreased anti-apoptotic $\mathrm{Bcl} 2, \mathrm{Bcl}-\mathrm{xl}$ protein expression, caused degradation in mitochondrial membrane potential, and induced apoptosis in NSCLC, A549, and H1299 cells in 
vitro associated with activation of 9, 3 caspases, poly (ADP- ribose) polymerase (PARP) (Singh et al.,2011).

60 healthy volunteers aged 19-57 (16 females and 51 males) were given $480 \mathrm{ml}$ of grape juice daily for an additional 8 weeks to the daily diet and blood samples were obtained from these patients. DNA damage was measured using a single cell gel (comet) test with alkaline electrophoresis. A significant reduction in lymphocyte DNA damage and reduction in the number of ROS/photons by $15 \%$ were determined compared to the beginning of the study (Park et al., 2003)

It has been found that grape seed proanthocyanidins have the ability to inhibit the invasion of human cutaneous HNSCC cells by reversing the epithelial-mesenchymal transition process targeting EGFR expression, can act as free radical scavengers and help reduce reactive oxygen species (ROS) (Sun et al., 2011).

In another study, the combination of resveratrol, quercetin and catechin $(0.5,5$ or $20 \mu \mathrm{M})$ were found to significantly reduce cell proliferation, block in vitro cell cycle continuity and reduce primary tumor growth (Schlachterman et al., 2008). In a recent study it was demonstrated that resveratrol suppressed tumor growth and inhibited leiomyoma cells in vitro (Chen et al., 2019). Again in another study, resveratrol was shown to inhibit oxidative stress induced with aflatoxin B1 in bovine mammary epithelial cells (Zhou et al., 2019).

Our findings support the hypothesis that resveratrol, of the polyphenolic compounds found in molasses, restricts DMBA-induced oxidative stress possibly through a reduction in free radical levels. In the literature review, while there were studies on anticancer in the presence of resveratrol in fruits in grape and various fruit juice concentrates, there were no studies comparing the effects of grape molasses and resveratrol on the rat reproductive organ.

Understanding the preventing and improving mechanisms of ovarian-damage of resveratrol and grape molasses which is found at higher rates in some foods suggests that it will play an active role in preventing many diseases. It is known that in recent years there has been an increase in the interest of alternative herbal or local foods in order to prevent or reduce tissue damage caused by cancer or cancer treatments. For this reason, several studies have been conducted on the antioxidant properties of green tea containing catechin and its derivatives, red onion containing quercetin, apples, tomatoes containing lycopene, foods containing isoflavones such as soybean, chickpeas and lentils, broccoli and brussels sprouts containing indol-3-carbinol, pomegranate containing polyphenol and ellagic acid, and many food products containing selenium, vitamin $\mathrm{E}$ and $\mathrm{D}$, anthocyanin, sulforaphane, citylbin and resveratrol. In addition to the abovementioned food products, our study results suggest grape molasses containing resveratrol, also known as black miracle, as an important antioxidant source. By increasing the number of groups specified, the effects on the metabolism damage at different doses can be supported by further clinical trials.

\section{Conclusion}

It was supported by the findings that the molasses as one of the most important nutrient sources of Eastern and Central Anatolia played an effective role as an effective agent in both the protection and the healing process when its effect on the reproductive system was compared to its active ingredient, resveratrol.

\section{Acknowledgements}

We thanks for all collaboration and contribution with laboratory personnel and Research Center of Experimental Animal Laboratory in Inonu University.

Ethics Committee Approval: Ethics committee approval was received for this study from Clinical Research Ethics Committee of Inonu University (2011/A-106).

Peer-review: Externally peer-reviewed.

Author Contributions: Externally peer-reviewed. Author Contributions: TRK, ABK Design TRK, ABK Supervision TRK, OO; Materials -TRK, ABK; Data Collection and/or Processing - TRK, $\mathrm{OO}, \mathrm{EK}, \mathrm{ABK}$; Analysis and/or Interpretation TRK, OO, EK, ABK; Literature Review - TRK, OO, EK, ABK; Writing - TRK, OO, EK, ABK; Critical Review - TRK, OO, EK, ABK.

Conflict of Interest: No conflict of interest was declared by the author.

Financial Disclosure: The authors declared that this study hasn't received no financial support. 


\section{References}

Aggarwal BB, Bhardwaj A, Aggarwal RS, Seeram NP, Shishodia S, Takada Y. Role of resveratrol in prevention and therapy of cancer: preclinical and clinical studies. Anticancer Research. 2004; 24: 2783-2840.

Al Fatease A, Shah V, Nguyen DX, Cote B, LeBlanc N, Rao DA, Alani AW. Chemosensitization and mitigation of Adriamycin-induced cardiotoxicity using combinational polymeric micelles for codelivery of quercetin/resveratrol and resveratrol/curcumin in ovarian cancer. Nanomedicine. 2019; 19: 39-48.

Armstrong BG, Hutchinson E, Unwin J, Fletcher T. Lung Cancer Risk after Exposure to Polycyclic Aromatic Hydrocarbons: A Review and MetaAnalysis. Environ Health Perspect. 2004; 112: 970-978.

Batu A, Kucuk E, Cimen M. Determination of the Physicochemical and Biochemical Values of Flower Honeys Obtained from Eastern Anatolia and Eastern Black Sea Regions. The Association of Food Technology. 2013; 8: 52-62.

Chanvitayapongs S, Draczynska - Lusiak B, Sun AY. Amelioration of oxidative stress by antioxidants and resveratrol in PC12 cells. Neuro Report. 1997; 8: 1499-1502.

Chen HY, Lin PH, Shih YH, Wang KL, Hong YH, Shieh TM, et al. Natural Antioxidant Resveratrol Suppresses Uterine Fibroid Cell Growth and Extracellular Matrix Formation In Vitro and In Vivo. Antioxidants (Basel). 2019; 8: 99.

Cortas NK, Wakid NW. Determination of inorganic nitrate in serum and urine by a kinetic cadmium reduction method. Clin Chem. 1990; 36: 14401443.

David H. Phillips. Polycyclic aromatic hydrocarbons in the diet. Mutation Research. 1999; 443:139-147.

De-la-Lastra CA, Villegas I. Resveratrol as an antiinflammatory and anti-aging agent: Mechanisms and clinical implications. Molecular Nutrition \& Food Research. 2005; 49: 405-430.

De La Lastra CA, Villages I. Resveratrol as an antioxidant and pro-oxidant agent: mechanisms and clinical implications. Biochemsoc trans. 2007; 35:1156-1160.

Ebeler SE, Brenneman CA, Kim GS, Jewell WT, Webb MR, Leticia CR, et al. Dietary catechin delays tumor onset in a transgenic mouse model. American Journal of Clinical Nutrition. 2002; 76: 865-872.
Eng ET, Ye J, Williams D, Phung S, Moore RE, Young MK, et al. Suppression of estrogen biosynthesis by procyanidin dimers in red wine and grape seeds. Cancer Res. 2003; 63: 85168522.

Francischi JN, Frade TIC, Almeida MPA, Queiroz BFG, Bakhle YS. "Ketamine-xylazine anaesthesia and orofacial administration of substance P: a lethal combination in rats," Neuropeptides. 2017; 62: 21-26.

Gao J, Lauer FT, Mitchell LA, Burchiel SW. Microsomal Expoxide Hydrolase Is Required for 7,12-Dimethylbenz[a]anthracene (DMBA)Induced Immunotoxicity in Mice. Toxicological Sciences. 2007; 98: 137-144.

Garvin S, Ollinger K, Dabrosin C. Resveratrol induces apoptosis and inhibits angiogenesis in human breast cancer xenografts in vivo. Cancer Letters. 2006; 231: 113-122.

Giovanni DJ, Juchau MR. Biotransformation and bioactivation of 7, 12 dimethylbenz (a) anthracene (7,12-DMBA). Drug Metab. 1980; 11: 61-101.

Im J, Kim H, Kim B, Yun J, Lee J, Lee C. A study on the characteristics of pollutant release and transfer registers (PRTRs) and cancer incidence rates in Korea. Environ Sci Pollut Res Int. 2019; 26: 17080-17090.

Jung KJ, Wallig MA, Singletary KW. Purple grape juice inhibits 7, 12- dimethylbenz [a] anthracene (DMBA)-induced rat mammary tumorigenesis and in vivo DMBA-DNA adduct formation. Cancer Lett. 2006; 233:279-288.

Katiyar SK. Grape seed proanthocyanidins and skin cancer prevention: inhibition of oxidative stress and protection of immune system. Molecular Nutrition and Food Research. 2008; 52: 71-76.

Kijima I, Phung S, Hur G, Kwok SL, Chen S. Grape seed extract is an aromatase inhibitor and a suppressor of aromatase expression. Cancer Res. 2006; 66: 5960-5967.

Kim H, Hall P, Smith M, Kirk M, Prasain JK, Barnes S, et al. Chemoprevention by Grape Seed Extract and Genistein in Carcinogen induced Mammary Cancer in Rats Is Diet Dependent. International Research Conference on Food, Nutrition and Cancer J Nutr. 2004; 134: 34453452.

Krishna PL, Pezzuto B, Pezzuto JM. Cancer Chemopreventive Activity of Resveratrol. Annals New York Academy Science. 2002; 957:210-229. 
Leonard SS, Xia C, Jiang BH, Stinefelt B, Klandorf H, Harris GK, Shi X. Resveratrol scavenges reactive oxygen species and effects radicalinduced cellular responses. Biochem Biophys Res Commun. 2003; 309:1017-1026.

Lopez-Oliva ME, Agis-Torres A, Goni I, Munoz Martinez E. Grape antioxidant dietary fibre reduced apoptosis and induced a pro-reducing shift in the glutathione redox state of the rat proximal colonic mucosa. British Journal of Nutrition. 2010; 103: 1110-1117.

Pacheco de Jesus GP, Ribeiro FAP, Gomes de Moura CF, Gollucke APB, Oshima CTF, Ribeiro DA. Anti-tumor activity of grape juice concentrate in the rat tongue two-stage initiation-promotion protocol induced by 4 nitroquinoline 1-oxide. Toxicol Mech Methods. 2014; 24: 276.

Park YK, Park E, Kim JS, Kang MH. Daily grape juice consumption reduces oxidative DNA damage and plasma free radical levels in healthy Koreans. Mutation Research. 2013; 529: 77-86.

Pharm RPB, Sahni JK, Ali J, Sharma S, Baboota S. Resveratrol: review on the rapeutic potential and recent advances in drug delivery. Expert Opin Drug Deliv.2014; 11: 1285-1298.

Santos AC, Pereira I, Magalhães M, Pereira-Silva M, Caldas M, Ferreira L, Figueiras A, Ribeiro AJ, Veiga F. Targeting Cancer Via ResveratrolLoaded Nanoparticles Administration: Focusing on In Vivo Evidence. The AAPS Journal. 2019; 21: 57.

Schlachterman A, Valle F, Wall KM, Azios NG, Castillo L, Morell L et al. Combined resveratrol, quercetin, and catechin treatment reduces breast tumor growth in a nude mouse model. Translational Oncology. 2008; 1: 19-27.

Shafiee M, Carbonneau MA, Urban N, Descomps B, Leger CL. Grape and grape seed extract capacities at protecting LDL against oxidation generated by $\mathrm{Cu}^{2+}$, AAPH or SIN1 and at decreasing superoxide THP-1 cell production. A comparison to other extracts or compounds. Free Radical Research. 2003; 37: 573-584.

Singh T, Sharma SD, Katiyar SK. Grape proanthocyanidins induce apoptosis by loss of mitochondrial membrane potential of human non-small cell lung cancer cells in vitro and in vivo. Plos One. 2011; 6: e27444
Souza CF, Baldissera MD, Descovi SN, Zeppenfeld CC, Verdi CM, Santos RCV, et al. Grape pomace flour alleviates Pseudomonas aeruginosa-induced hepatic oxidative stress in grass carp by improving antioxidant defense. Microb Pathog. 2019;129:271-276.

Sun Q, Prasad R, Rosenthal E, Katiyar SK. Grape seed proanthocyanidins inhibit the invasive potential of head and neck cutaneous squamous cell carcinoma cells by targeting EGFR expression and epithelial-to-mesenchymal transition. BMC Complementary and Alternative Medicine. 2011; 11: 134.

Tietze F, Enzymic method for quantitative determination of nanogram amounts of total and oxidized glutathione: applications to mammalian blood and other tissues. Anal Biochem. 1969; 27: 502-522.

Truong VL, Jun M, Jeong WS. Role of resveratrol in regulation of cellular defense systems against oxidative stress. BioFactors. 2018; 44: 36-49.

Uchiyama M, Mihara M. Determination of malonaldehyde precursor in tissues by thiobarbituric acid test. Anal Biochem. 1978; 86: 271-278.

Ustun MS, Tosun I. The Composition of Pekmez. The JOurnal of Foodç 1997; 22: 417-423.

$\mathrm{Yu} \mathrm{Z}, \mathrm{Xu} \mathrm{W}$, Wang $\mathrm{H}$. Resveratrol treatment inhibits acute pharyngitis in the mice model through inhibition of PGE2/COX-2 expression. Saudi J Biol Sci. 2018; 25: 1468-1472.

Zadi Heydarabad M, Nikasa M, Vatanmakanian M, Azimi A, Farshdousti Hagh M. Regulatory effect of resveratrol and prednisolone on MDR 1 gene expression in acute lymphoblastic leukemia cell line (CCRF-CEM): An epigenetic perspective. J Cell Biochem. 2018; 119: 4890-4896.

Zeweil MM, Sadek KM, Taha NM, El-Sayed Y, Menshawy S. Graviola attenuates DMBAinduced breast cancer possibly through augmenting apoptosis and antioxidant pathway and downregulating estrogen receptors. Environ Sci Pollut Res Int. 2019; 26: 150209-150217.

Zhao J, Wang J, Chen Y, Agarwal R. Anti-tumorpromoting activity of a polyphenolic fraction isolated from grape seeds in the Mouse skin twostage initiation-promotion protocol and identification of procyanidin B5-3' -gallate as the most effective antioxidant constituent. Carcinogenesis. 1999; 20: 1737-1745. 
Zheng X, Jia B, Song X, Kong QY, Wu ML, Qiu $\mathrm{ZW}$, Li H, Liu J. Preventive Potential of Resveratrol in Carcinogen-Induced Rat Thyroid Tumorigenesis. Nutrients. 2018; 10: 279.

Zhou Y, Jin Y, Yu H, Shan A, Shen J, Zhou C, et al. Resveratrol inhibits aflatoxin B1-induced oxidative stress and apoptosis in bovine mammary epithelial cells and is involved the Nrf2 signaling pathway. Toxicon. 2019; 164: 1015. 